\title{
Calibration of Mineralization Degree for Dynamic Pure-water Measurement in Horizontal Oil-water Two-phase Flow
}

\author{
Weihang Kong ${ }^{1}$, Lei $\mathrm{Li}^{1,3}$, Lingfu Kong ${ }^{1,2}$ and Xingbin Liu $^{3}$ \\ ${ }^{1}$ School of Information Science and Engineering, Yanshan University, Qinhuangdao 066004, China, kongweihang@163.com \\ ${ }^{2}$ Computer Virtual Technology \& System Integration Key Laboratory of Hebei Province, Qinhuangdao 066004, China \\ ${ }^{3}$ Logging and Testing Services Company, Daqing Oilfield Limited Company, Daqing 163453, China
}

\begin{abstract}
In order to solve the problem of dynamic pure-water electrical conductivity measurement in the process of calculating water content of oilwater two-phase flow of production profile logging in horizontal wells, a six-group local-conductance probe (SGLCP) is proposed to measure dynamic pure-water electrical conductivity in horizontal oil-water two-phase flow. The structures of conductance sensors which include the SGLCP and ring-shaped conductance probe (RSCP) are analyzed by using the finite-element method (FEM). In the process of simulation, the electric field distribution generated by the SGLCP and RSCP are investigated, and the responses of the measuring electrodes are calculated under the different values of the water resistivity. The static experiments of the SGLCP and RSCP under different mineralization degrees in horizontal oil-water two-phase flow are carried out. Results of simulation and experiments demonstrate a nice linearity between the SGLCP and RSCP under different mineralization degrees. The SGLCP has also a good adaptability to stratified flow, stratified flow with mixing at the interface and dispersion of oil in water and water flow. The validity and feasibility of pure-water electrical conductivity measurement with the designed SGLCP under different mineralization degrees are verified by experimental results.
\end{abstract}

Keywords: Horizontal oil-water two-phase flow, mineralization degree, local-conductance probe (LCP), pure-water measurement.

\section{INTRODUCTION}

With the rapid development of horizontal well technology, water content of the whole well will increase sharply when the part is flooded in the process of horizontal well production and development, which has a negative impact on development effectiveness, even leads to the waste of oil well [1], [2]. Thus, the horizontal producing profile well logging is becoming more and more important, in particular, water content which is one of the important parameters of multiphase flow detection in oilfield logging, and there is an urgent need for accurate measurement in horizontal oil field production.

At the beginning of oilfield development, the water content was relatively low [3]. The method of water content measurement has improved to some degree by using concentric overcurrent capacitive sensor [4], [5]. With the continuous development of oil fields, most oil wells have gradually entered high water content stage because of longterm development with water injection [6]. Meanwhile, high deviated and horizontal well technology is also being increasingly adopted [7], [8]. In such a complex condition, concentric overcurrent capacitance sensor immersed in pureoil or pure-water sometimes results in a lower resolution and a greater error, and it cannot meet the needs of the actual measurement [9], [10]. The principle of conductance water cut meter (CWCM), including both the ring-shaped conductance probe (RSCP) for pure-water electrical conductivity measurement and RSCP for oil-water mixture electrical conductivity measurement, is based on the Maxwell formula [11], when the fluid is a continuous water phase, and the water holdup can be determined by the ratio of the oil-water mixture electrical conductivity and purewater electrical conductivity [12], [13]. It has been widely used in vertical production profile logging in terms of water holdup measurement [14]-[16].

However, in horizontal production profile logging, the oilwater two-phase flow patterns of long distance undulating horizontal well have a mixed flow pattern which is mainly a horizontal segregated flow as opposed to vertical well [17], [18], and flow pattern and velocity are influenced by the inclination of long distance undulating horizontal well, that leads to the pre-existing ring-shaped measuring electrodes of the CWCM being still immersed in oil-water segregated flow when sampling. Although oil-water mixture electrical conductivity could be measured on-line in the process of fluid flow by using the CWCM [19], pure-water phase conductivity could not be obtained by using the ring-shaped electrodes or with a sampler at the condition of oil-water two-phase separation. So it is impossible to carry out purewater result sampling calibration, and the pre-existing CWCM cannot be applied in horizontal wells in term of horizontal well structure and ring-shaped electrode structure of the CWCM. 
Meanwhile, electrical conductivity reflects the concentration content in water conductive ions, the greater is the concentration of conductive ions in water, the stronger is electrical conductivity. Mineralization degree is the total amount of inorganic mineral elements contained in the water. Therefore, the concentration of mineralization degree determines the degree of the fluid electrical conductivity. According to the electrical principle [13], it is known that the voltage amplitude between two measuring electrodes of the conductance sensor is inversely proportional to the electrical conductivity of the fluid, and the degree of the fluid electrical conductivity depends on the concentration of the fluid mineralization degree. Currently, common tap water mineralization degree content is less than 1000 parts per million (ppm) and the original formation water mineralization degree is about $6000 \mathrm{ppm}$. Increased oil recovery is caused by water injection changes in the produced water because the injected water and the formation water have different mineralization degree [20], [23]. Therefore, the value of mineralization degree of oil field water varies from $2000 \mathrm{ppm}$ to $3000 \mathrm{ppm}$. The change of mineralization degree will lead the oil-water mixture electrical conductivity and pure-water electrical conductivity measured by conductance probe to increase or decrease proportionally to maintain relatively constant water content [24]. Because the fluid mineralization degree in production profile logging is very different from that in the simulation well, the pure-water calibration result of the simulation well cannot calculate the water content in oil well.

Due to the horizontal well casing, tubing and measuring instruments are of an axisymmetric cylindrical structure and the measuring instruments can only rely on gravity or the horizontal crawler reaching the downhole oil-layer measuring section. Consequently, it is impossible to determine the work position of the measuring instruments after the axial rotation. Simultaneously, single sensor is greatly influenced by the fluid flow pattern [25], [26], so it is required that the sensor design model is an axisymmetric array structure, so as to reduce the influence of the flow pattern and the error caused by the axial rotation. In this paper, the SGLCP is designed for dynamic pure-water measurement of oil-water two-phase segregated flow in horizontal pipes. The calibration of simulation and experiments under different mineralization degrees of water were carried out by using both SGLCP and RSCP, and verified the validity of pure-water electrical conductivity measurement with the new SGLCP by experimental results.

\section{THE GEOMETRY OF CONDUCTANCE PROBES}

\section{A. The geometry of the SGLCP}

The geometry of the SGLCP for pure-water measurement is shown in Fig.1. The SGLCP consists of six pairs of metal parallel-electrodes, in which each pair of metal parallelelectrodes is a local-conductance probe (LCP), and includes an exciting electrode and measuring electrode along the axial section direction. In addition, one end of each electrode flush-mounted on the inside wall of the insulated flow pipe, six electrodes represented by $E_{1}$ to $E_{6}$ are homogeneously distributed on the same upstream cross section along the axial direction, and six electrodes represented by $M_{1}$ to $M_{6}$ are homogeneously distributed on the same downstream cross section along the axial direction. Each of $E_{i}$ and $M_{i}$ is exciting electrode and measuring electrode simultaneously, and $\mathrm{E}_{i}$ and $\mathrm{M}_{i}$ electrodes formed the $i$-th $\mathrm{LCP}_{i}$, where the range of $i$ is from 1 to 6 .

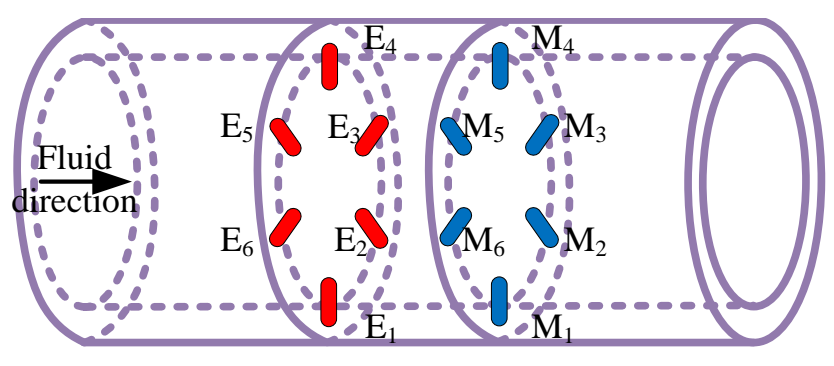

Fig.1. Geometry of the SGLCP for dynamic pure-water measurement.

The representing structure parameters and coordinates of the SGLCP are shown in Fig.2., in which $S$ is the separation between two electrodes of the $\mathrm{LCP}_{i}, d$ is the diameter of the metal parallel-electrode, $h$ is the measurement field height of the metal parallel-electrode, $D$ is the inner diameter of the insulated flow pipe, $D_{w}$ is the thickness of the insulated flow pipe, and $L$ is the axial length of the insulated flow pipe.

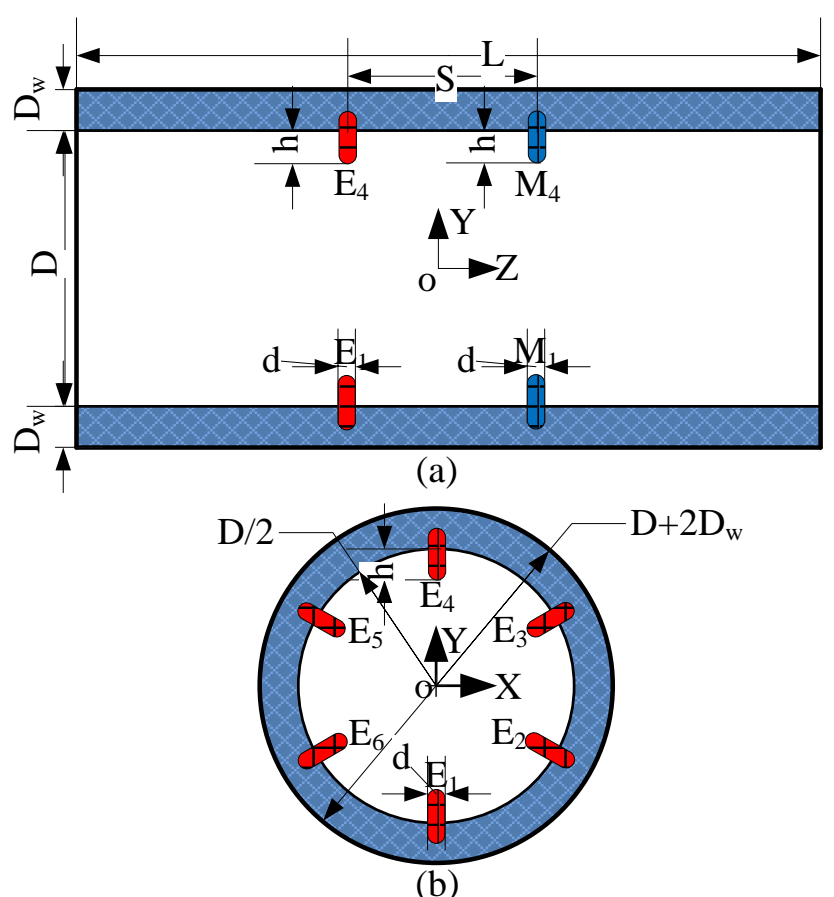

Fig.2. Sketches of structure parameters and coordinates of the SGLCP: a) The axial section; b) The cross section. 


\section{B. The geometry of the RSCP}

The RSCP [14] includes four ring-shaped stainless steel electrodes which are embedded in the insulating wall with a certain distance in fluid pipeline. There are two ring-shaped exciting electrodes on both sides of the sensor to provide an alternating constant current. The two ring-shaped electrodes in the middle are used for measurement. Due to the presence of the impedance between the electrodes, when alternating constant current runs through ring-shaped measuring electrodes by using the conductive fluid, the voltage signal is generated by two measuring electrodes, the amplitude of which is inversely proportional to the fluid electrical conductivity through the RSCP based on electrical principle.

The structure parameters and coordinates of the RSCP are shown in Fig.3., in which $S_{\mathrm{e}}$ is the distance between exciting electrodes, $h_{\mathrm{e}}$ is the height of exciting electrodes, $S_{\mathrm{m}}$ is the distance between the measuring electrodes, $h_{\mathrm{m}}$ is the height of measuring electrodes, $D$ is the inner diameter of the insulated flow pipe, $D_{\mathrm{w}}$ is the thickness of the insulated flow pipe, $L_{\mathrm{e}}$ is the axial length of the insulated flow pipe. The probe consists of four stainless steel ringshaped electrodes axially separated and flush-mounted on the inside wall of the insulated flow pipe. E1 and E2 represent exciting electrodes, M1 and M2 represent the measuring electrodes.
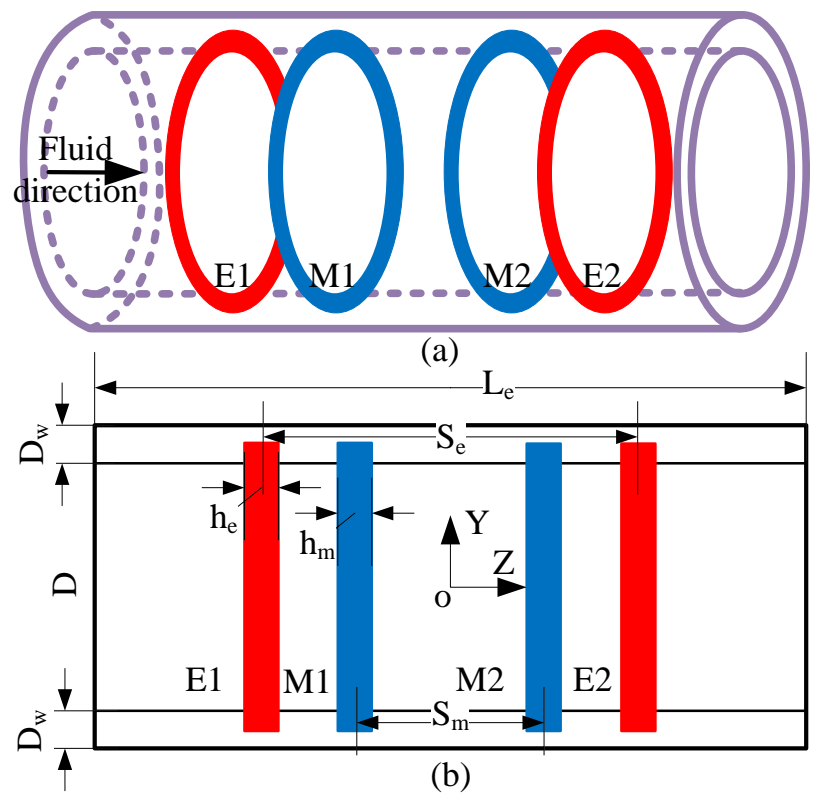

Fig.3. Geometry and parameter definition of the RSCP: a) 3D model; b) 2D structure.

The polarization phenomenon can occur in the vicinity of the exciting electrodes when the SGLCP or RSCP is excited by a low frequency electrical power source, which can result in parasitic impedance and a detectable measuring error. In order to avoid the polarization phenomenon, we adopt an alternating voltage source as the exciting signal in the actual application. Besides, the two exciting electrodes are connected with a sinusoidal $20 \mathrm{kHz}$ exciting signal in the measurement system, and the response signals from measuring electrodes of the SGLCP and RSCP are correlated with the dynamic pure-water electrical conductivity of oil-water two-phase flow for a certain given mineralization degree in horizontal pipes.

\section{NUMERICAL SIMULATION OF CONDUCTANCE PROBES}

\section{A. The meshed model and electric field distribution of the SGLCP}

Since the measurement field of the SGLCP is much smaller than the wavelength of the electric field, the electric field of the SGLCP can be modeled as time invariant. Regarding this, a direct current source was used as the exciting signal to investigate the electric field of the SGLCP. The distribution of the inner electric field of the SGLCP can be described by the Laplace equation with some certain boundary conditions under the cylindrical coordinates [27]

$$
\nabla^{2} u(r, \varphi, z)=\frac{1}{r} \frac{\partial}{\partial r}\left(r \frac{\partial u}{\partial r}\right)+\frac{1}{r^{2}} \frac{\partial^{2} u}{\partial \varphi}+\frac{\partial^{2} u}{\partial z^{2}}=0
$$

Each $\mathrm{LCP}_{i}$ of the SGLCP is constrained by the essential boundary conditions, which can be expressed as

$$
\left\{\begin{array}{l}
u=0\left(r \in\left[\frac{D}{2}-h, \frac{D}{2}\right],\right. \\
\varphi \in\left[\frac{i-1}{3} \pi+\arctan \left(\frac{d}{D-2 h}\right), \frac{i}{3} \pi-\arctan \left(\frac{d}{D-2 h}\right)\right], \\
\left.-\frac{L}{2} \leq z \leq-\frac{S+d}{2},-\frac{S-d}{2} \leq z \leq \frac{S-d}{2}, \frac{S+d}{2} \leq z \leq \frac{L}{2}\right) \\
\frac{\partial u}{\partial r}=\frac{I_{e}}{S_{d}}\left(r \in\left[\frac{D}{2}-h, \frac{D}{2}\right],\right. \\
\varphi \in\left[\frac{i-1}{3} \pi-\arctan \left(\frac{d}{D-2 h}\right), \frac{i-1}{3} \pi+\arctan \left(\frac{d}{D-2 h}\right)\right] \\
\left.\frac{S-d}{2} \leq z \leq \frac{S+d}{2},-\frac{S+d}{2} \leq z \leq-\frac{S-d}{2}\right) \\
\frac{\partial u}{\partial z}=0\left(z= \pm \frac{L}{2}\right)
\end{array}\right.
$$

where $u$ is the inner electric potential distribution, $I_{e}$ represents the direct current applied to the exciting current, $S_{d}$ is the surface area of the electrodes of the $\mathrm{LCP}_{i}$.

The FEM is employed to investigate the distribution of the electric field inside the SGLCP. Fig.4. shows the meshed finite element model of the SGLCP in ANSYS software in which we chose SOLID232 as the element type and used free meshing to divide the model. The total element number of the meshed finite model is 250295. The direct current of $0.1 \mathrm{~mA}$ is supplied to $\mathrm{E}_{i}$, the current of $-0.1 \mathrm{~mA}$ is supplied to $\mathrm{M}_{i}$ and the voltage of electrode $\mathrm{M}_{i}$ is set to $0 \mathrm{~V}$. 


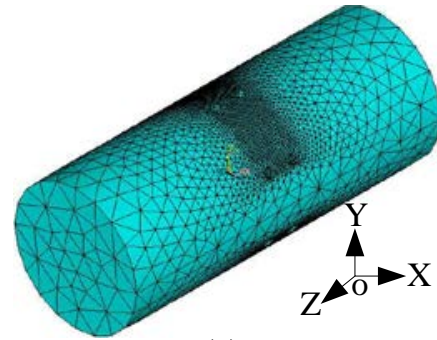

(a)

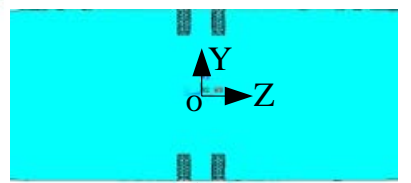

(b)
Fig.4. The meshed 3D model of the SGLCP with 250295 elements: $\mathrm{L}=0.05 \mathrm{~m}, \mathrm{D}=0.02 \mathrm{~m}, \mathrm{~h}=0.002 \mathrm{~m}, \mathrm{~d}=0.0015 \mathrm{~m}$, $\mathrm{S}=0.004 \mathrm{~m}$, water resistivity $\sigma_{w}=100 \Omega \mathrm{m}:$ a) 3D model; b) The axial section.

The electric potential distribution of the SGLCP is shown in Fig.5. As can be seen from Fig.5., the electric potential between the exciting electrodes presents a high gradient, and the potential in the position away from the exciting electrodes tends to change slowly. Distribution of the electric field of the SGLCP is shown in Fig.6., from which it can be seen that the electric field between the exciting electrodes presents a stronger and homogeneous distribution; away from the exciting electrodes electric field is weaker and tends to 0 .

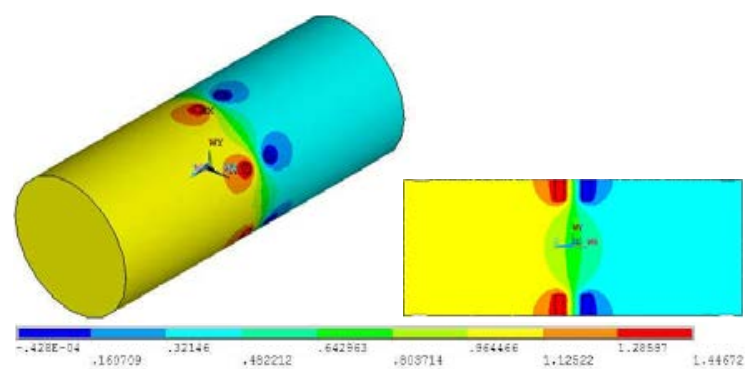

(a)

(b)

Fig.5. Electric potential distribution of the SGLCP: a) 3D view; b) The axial section.
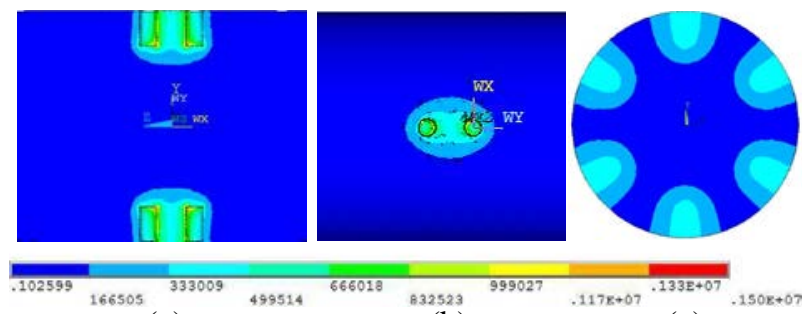

(a)

(b)

(c)

Fig.6. Electric field distribution of the SGLCP: a) YoZ plane section view; b) XoZ plane section view; c) $\mathrm{XoY}$ plane section view.

\section{$B$. The meshed model and electric field distribution of the $R S C P$}

As with above, the measurement field of the RSCP is much smaller than the wavelength of the electric field, the electric field of the RSCP can be modeled as time invariant. In this regard, we use a direct current source as the exciting signal to investigate the electric field of the RSCP. The inner electric field distribution of the RSCP can be described by the Laplace equation with some certain boundary conditions under the cylindrical coordinates [27]

$$
\nabla^{2} u(r, \varphi, z)=\frac{1}{r} \frac{\partial}{\partial r}\left(r \frac{\partial u}{\partial r}\right)+\frac{1}{r^{2}} \frac{\partial^{2} u}{\partial \varphi}+\frac{\partial^{2} u}{\partial z^{2}}=0
$$

The essential boundary conditions can be expressed as

$$
\begin{aligned}
& u=0\left(r=\frac{D}{2}, \frac{S_{e}+h_{e}}{2} \leq z \leq \frac{L_{e}}{2},\right. \\
& \left.-\frac{L_{e}}{2} \leq z \leq-\frac{S_{e}+h_{e}}{2},-\frac{S_{e}-h_{e}}{2} \leq z \leq \frac{S_{e}-h_{e}}{2}\right) \\
& \left\{\frac{\partial u}{\partial r}=\frac{I_{e}}{S_{R}}\left(r=\frac{D}{2}, \frac{S_{e}-h_{e}}{2} \leq z \leq \frac{S_{e}+h_{e}}{2},\right.\right. \\
& \left.-\frac{S_{e}+h_{e}}{2} \leq z \leq-\frac{S_{e}-h_{e}}{2}\right) \\
& \frac{\partial u}{\partial z}=0\left(z= \pm \frac{L_{e}}{2}\right)
\end{aligned}
$$

where $u$ is the inner electric potential distribution, $D$ is the inner diameter of the insulated flow pipe, $L_{e}$ is the insulated flow pipe length, $h_{e}$ is the height of exciting electrodes, and $S_{e}$ is the separation between two exciting electrodes, $I_{e}$ represents the direct current applied to the exciting current and $S_{R}$ is the surface area of the exciting electrodes of the $R S C P$. The FEM were employed to investigate the electric field distribution inside the RSCP. Fig.7. shows the meshed finite element model of the RSCP in ANSYS software in which we chose SOLID232 as the element type and divided the model by free meshing. The total element number of the meshed finite model is 10692. The direct current of $0.1 \mathrm{~mA}$ is supplied to the exciting electrodes E1, the current of $0.1 \mathrm{~mA}$ is supplied to E2 and at the same time the voltage of electrode $\mathrm{E} 2$ is set to $0 \mathrm{~V}$.

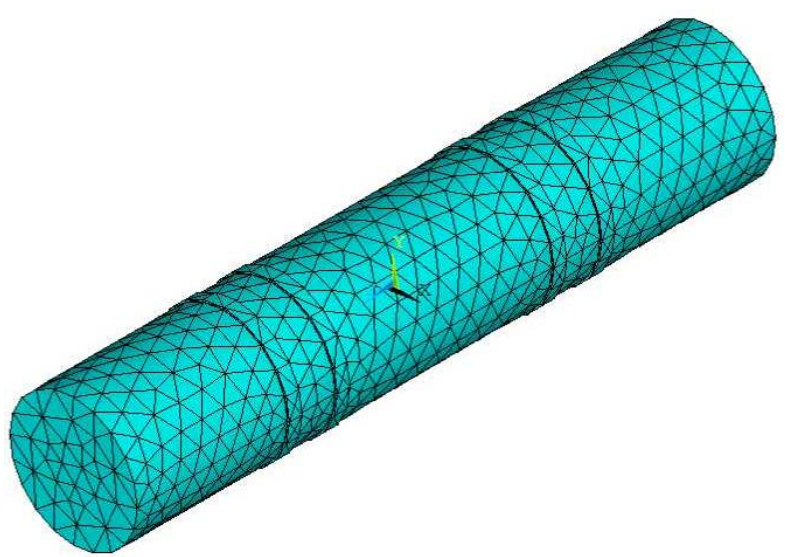

Fig.7. The meshed 3D model of the RSCP with 10692 elements: $L_{e}=0.10 \mathrm{~m}, D=0.02 \mathrm{~m}, h_{e}=0.002 \mathrm{~m}, h_{\mathrm{m}}=0.002 \mathrm{~m}, S_{e}=0.047 \mathrm{~m}$, $S_{\mathrm{m}}=0.032 \mathrm{~m}, \sigma_{w}=100 \Omega \mathrm{m}$. 
The calculated result of the RSCP electric field is shown in Fig.8. As can be seen from Fig.8., the electric potential between the exciting electrodes presents a high gradient, away from the exciting electrodes the electric potential tends to change slowly. The electric field between the measuring electrodes presents a stronger and homogeneous distribution, the electric field between the measuring electrode and exciting electrode presents an inhomogeneous distribution and away from the exciting electrodes electric field is weaker and tends to 0 .

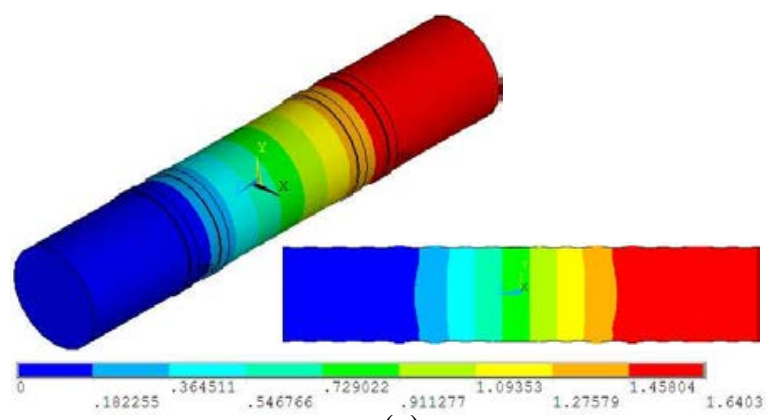

(a)

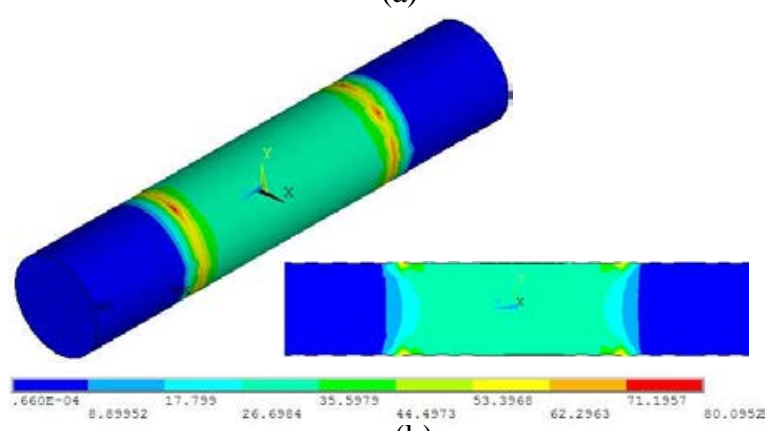

(b)

Fig.8. Electric field distribution of the RSCP with $\sigma_{w}=100 \Omega \mathrm{m}$ : a) Electric potential; b) Electric field.

\section{CALCULATED RESPONSE OF SIMULATION FOR} CONDUCTANCE PROBES UNDER DIFFERENT WATER RESISTIVITY

\section{A. Calculated response of the SGLCP}

In this study, in order to measure the pure-water electrical conductivity in the condition of the horizontal oil-water segregated flow, due to the location of the LCP at the bottom of the insulated pipe and the phenomenon that the underlayer presents a continuous water phase and the upperlayer presents the oil phase or oil-water phase in horizontal oil-water two-phase flow, the investigation can only be on the response characteristics of the $\mathrm{LCP}_{1}$ in the SGLCP, and the analysis of the feasibility and working conditions for the $\mathrm{LCP}_{1}$ to obtain the pure-water electrical conductivity is required.

The models for different water holdup $y_{m}$ in which the geometry of the $\mathrm{LCP}_{1}$ was $D=20 \mathrm{~mm}, h=2 \mathrm{~mm}, d=1.5 \mathrm{~mm}$, $S=4,5,6 \mathrm{~mm}$, respectively were simulated. The calculated response of the $\mathrm{LCP}_{1}$ was analyzed in term of $y_{m}$, the range of $y_{m}$ was from 0.05 to 1 , and the change interval with 0.05 was used as an experimental point. The result curves of the simulation calculation are shown in Fig.9. $V_{m}$ is the measured voltage and can be calculated by

$$
V_{m}=k_{m} \times \sigma_{m} \times I_{\mathrm{e}}
$$

where $k_{m}$ is resistance correction coefficient, $\sigma_{m}$ represents oil-water mixture electrical resistivity, $I_{\mathrm{e}}$ represents exciting current.

As can be seen from Fig.9., under the conditions of higher $y_{m}$, the $\mathrm{LCP}_{1}$ can obtain a reliable and accurate pure-water electrical conductivity, which can be used as the pure-water correction value for water content measurement in horizontal oil water segregated flow. And in the condition of the $\mathrm{LCP}_{1}$ with $D=20 \mathrm{~mm}, h=2 \mathrm{~mm}, d=1.5 \mathrm{~mm}, S=4,5,6 \mathrm{~m}$, respectively, the response result is approximately equal to pure-water electrical conductivity when $y_{m}$ is greater than or equal to 0.30 .

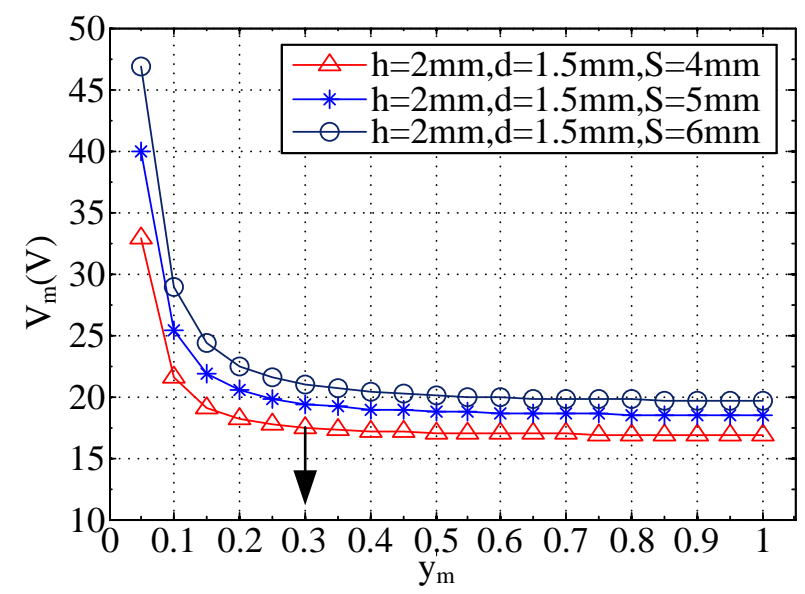

Fig.9. Calculated response of the simulation for $\mathrm{LCP}_{1}$ under different values of $y_{m}: D=20 \mathrm{~mm}, \sigma_{w}=100 \Omega m$ and oil resistivity $\sigma_{o}=1.0 E+13 \Omega m$.

It can be known from Fig.9. that the SGLCP can obtain the pure-water electrical conductivity at the condition of high water holdup in horizontal oil-water two-phase segregated flow.

In order to investigate the response characteristics for the different mineralization degrees of water in the fluid to the SGLCP, the different values of the water electrical resistivity are used to represent the different mineralization degrees of water during the simulation. The response values of the simulation are calculated at the condition filled with a continuous water phase in the insulated pipe under different values of $\sigma_{w}$ in which the geometry of the SGLCP is $D=20 \mathrm{~mm}, h=2 \mathrm{~mm}, d=1.5 \mathrm{~mm}, S=4,5,6 \mathrm{~mm}$, respectively. The value range of $\sigma_{w}$ is from $100 \Omega \mathrm{m}$ to $2000 \Omega \mathrm{m}$ by using the finite element model of the SGLCP. 
The calculated response of the SGLCP for pure-water measurement under different values of $\sigma_{w}$ is shown in Fig.10., in which $V_{\mathrm{S}}$ is the voltage measured by the measuring electrodes of the SGLCP. As can be observed from Fig.10., in different conditions of $\sigma_{w}$, the response characteristics of the SGLCP, whose $S$ is set to three different values, are basically consistent. The response result $V_{\mathrm{S}}$ of the SGLCP is greatly influenced by the variation of $\sigma_{w}$ with the increase of $\sigma_{w}$, the response results $V_{\mathrm{S}}$ of the SGLCP with three different $S$ show linear increasing tendency, and show obvious difference which can be attributed to the distinctive difference of $\sigma_{w}$. Under the conditions of a certain given $\sigma_{w}$, the greater the $S$ is, the greater the response result $V_{\mathrm{S}}$ of the SGLCP becomes.

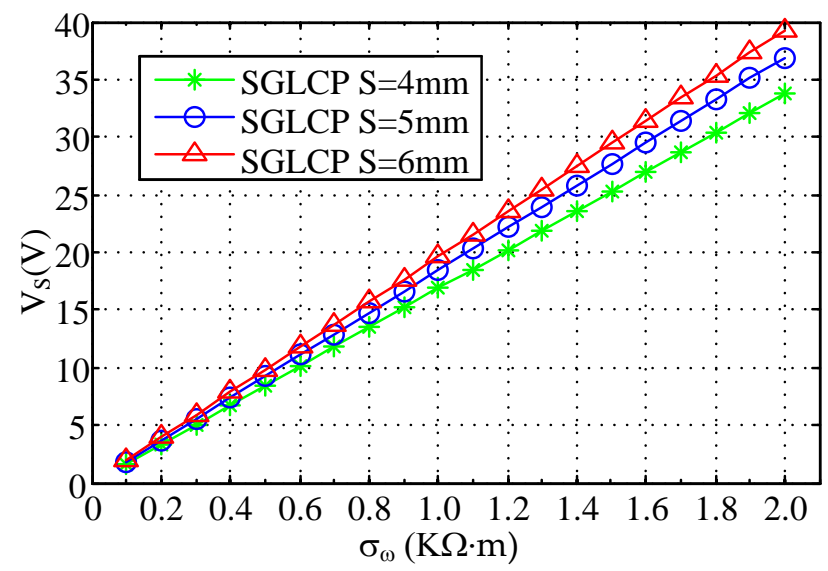

Fig.10. Calculated response of the simulation for the SGLCP under different values of $\sigma_{w}: h=2 \mathrm{~mm}, d=1.5 \mathrm{~mm}, D=20 \mathrm{~mm}$.

\section{B. Calculated response of the RSCP}

The calculated response of the RSCP for pure-water measurement under different values of $\sigma_{w}$ is calculated by using the finite element model of the RSCP. The simulated model for different values of $\sigma_{w}$ in which the geometry of the RSCP is $h_{\mathrm{e}}=2 \mathrm{~mm}, h_{\mathrm{m}}=2 \mathrm{~mm}, S_{\mathrm{e}}=47 \mathrm{~mm}, S_{\mathrm{m}}=32 \mathrm{~mm}$, the range of $\sigma_{w}$ is from $100 \Omega \mathrm{m}$ to $2000 \Omega \mathrm{m}$. The calculated response of the RSCP for pure-water measurement under different values of $\sigma_{w}$ is shown in Fig.11., in which $V_{\mathrm{R}}$ is the voltage measured by the measuring electrodes of the RSCP. As can be observed from Fig.11., the response result $V_{\mathrm{R}}$ of the RSCP is greatly influenced by the variation of $\sigma_{w}$, with the increase of $\sigma_{w}$, the $V_{\mathrm{R}}$ shows a linear increasing tendency, and shows obvious difference which can be attributed to the distinctive different $\sigma_{w}$.

According to Fig.10. and Fig.11., it is found that the SGLCP and RSCP have nice linearity characteristics for pure-water electrical conductivity measurement under different values of $\sigma_{w}$, respectively. The calibration curves of response results between the SGLCP and RSCP are shown in Fig.12. As can be observed from Fig.12., under the condition of different values of $S$, the response values between the SGLCP and RSCP show a linear function relation, and the calibration curves between the SGLCP and RSCP can be given as

$$
\left\{\begin{array}{l}
V_{\mathrm{S}}=1.0080 V_{\mathrm{R}}-0.00019, S=4 \mathrm{~mm} \\
V_{\mathrm{S}}=1.1035 V_{\mathrm{R}}-0.00001, S=5 \mathrm{~mm} \\
V_{\mathrm{S}}=1.1740 V_{\mathrm{R}}-0.00002, S=6 \mathrm{~mm}
\end{array}\right.
$$

where $V_{\mathrm{R}}$ is the measured voltage of the RSCP, $V_{\mathrm{S}}$ is the measured voltage of the SGLCP, $S$ is the separation between two measuring electrodes of the $\mathrm{LCP}_{i}$.

In theory, the constants in (6) should be 0 . In the process of simulation modeling, due to the effect the different numbers of the grid division for sensors with different structure parameters have on the response results, the constants in (6) are no longer 0 , which leads to a minor error.

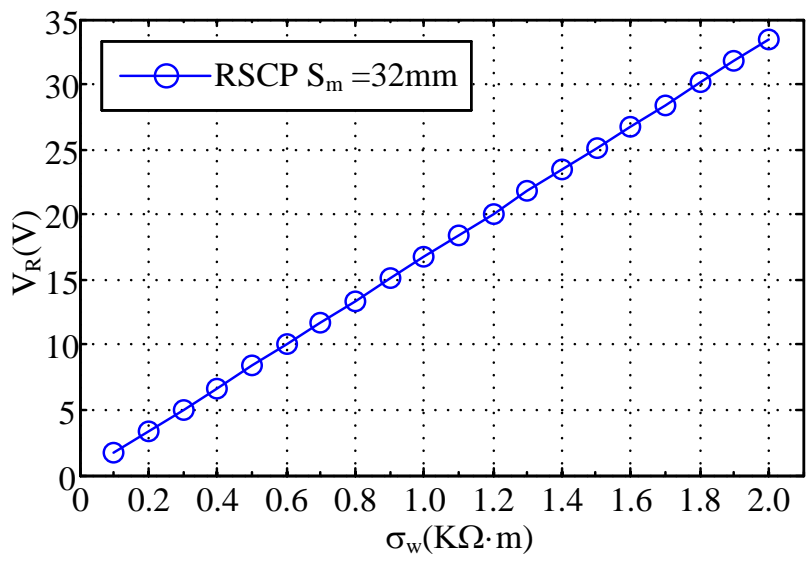

Fig.11. Calculated response of the simulation for the RSCP under different values of $\sigma_{w}: h_{\mathrm{e}}=2 \mathrm{~mm}, h_{\mathrm{m}}=2 \mathrm{~mm}, S_{\mathrm{e}}=47 \mathrm{~mm}$, $S_{\mathrm{m}}=32 \mathrm{~mm}$.

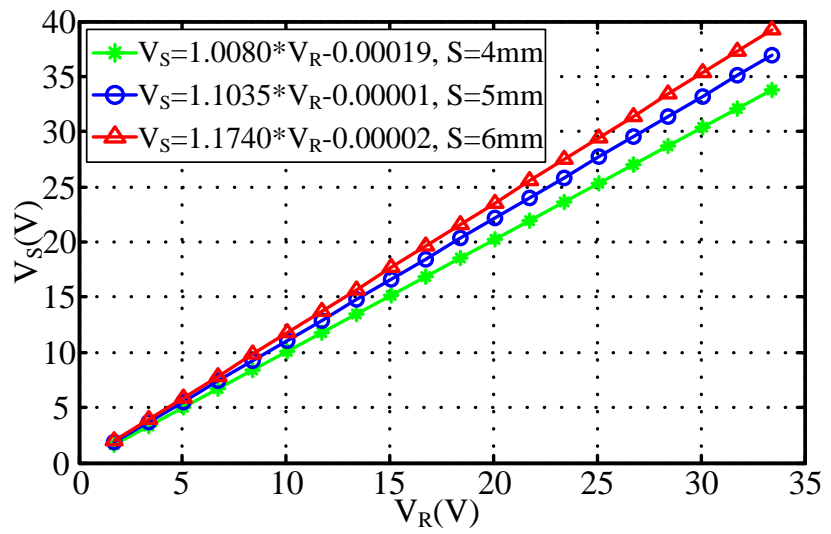

Fig.12. The calibration curves of simulation results between the SGLCP and RSCP. 


\section{STATIC EXPERIMENTS OF THE SGLCP AND RSCP UNDER DIFFERENT MINERALIZATION DEGREES}

For the SGLCP and RSCP, we adopt inhibit current loss, multiplex programmable analog switch circuit, differential amplifier, RMS to DC converter and V/F conversion measurement circuit and construct the pure-water measurement system. The instrument output is expressed by the frequency signal $F_{w}(\mathrm{KHz})$ which can be described as

$$
F_{w}=k_{w} \times V_{w} \times 10^{-3}
$$

where $k_{w}$ is correction coefficient, $V_{w}$ represents the measuring direct voltage for a certain given mineralization degree water.

Fig.13. shows the images of frequency measurement circuitry which includes multiplex programmable analog switch circuit, dual channel exciting signal circuit, DC power conversion circuit, dual channel differential amplifier, RMS to DC converter and V/F converter circuits, dual frequency signals complex circuit, and power generation circuit.

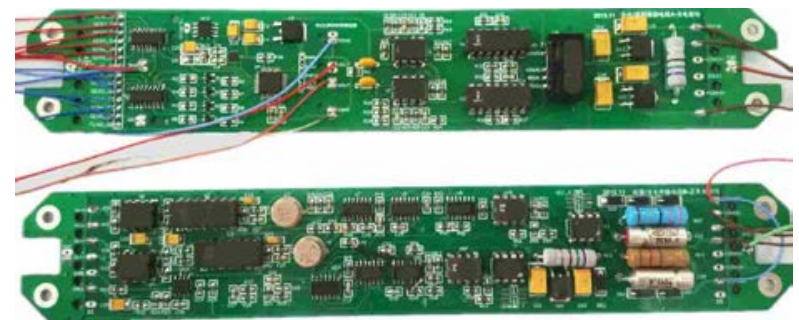

Fig.13. Images of frequency measurement circuitry for the SGLCP and RSCP.

Images of conductance probes are shown in Fig.14. which shows the structures of the SGLCP and RSCP with the axially separated, cross section distribution and flushmounted on the inside wall of the insulated flow pipe with the inner diameter of $20 \mathrm{~mm}$.

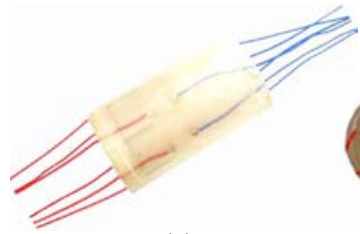

(a)

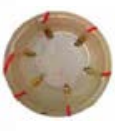

(b)

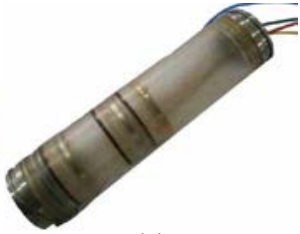

(c)
Fig.14. Images of conductance probes: a) The SGLCP; b) Cross section of the SGLCP; $c$ ) The RSCP.

Fig.15. shows horizontal oil-water two-phase flow experiment equipment in petrodaqing test \& experiment center of logging \& testing technology. To calibrate the SGLCP and RSCP for pure-water measurement under different mineralization degrees, the calibration test for different mineralization degrees of water is carried out.

Since the mineralization degree value of the downhole fluid ranges from $2000 \mathrm{ppm}$ to $3000 \mathrm{ppm}$, the downhole fluid is considered a dilute solution. And in dilute solution the conductance can be expressed as [28]:

$$
S_{c}=\lambda \cdot \frac{C}{\delta \cdot 1000} \cdot \frac{A}{l}
$$

where $S_{c}$ is the conductance of the solution (S), $\lambda$ is the equivalent conductivity of the solution $\left(\mathrm{S} \cdot \mathrm{cm}^{-2} \cdot \mathrm{g}^{-1}\right), \delta$ is the milliequivalent of the solution (mg), $A$ is the electrode area $\left(\mathrm{cm}^{2}\right), l$ represents the distance between two electrodes, and $C$ is the concentration of solution $(\mathrm{mg} / \mathrm{L})$.

In the formula (8), it shows that the conductance $S_{c}$ only depends on the concentration $C$. Let $F=\lambda \cdot \frac{1}{\delta \cdot 1000} \cdot \frac{A}{l}$, we get

$$
S_{c}=F \cdot C
$$

In this experiment $\lambda$ and $\delta$ of the salt-containing solution are both constants, so $F$ is also a constant. Therefore, there is a linear relation between the conductance $S_{c}$ and the concentration $C$, and the static experiment used the different salt concentrations to represent different mineralization degrees.

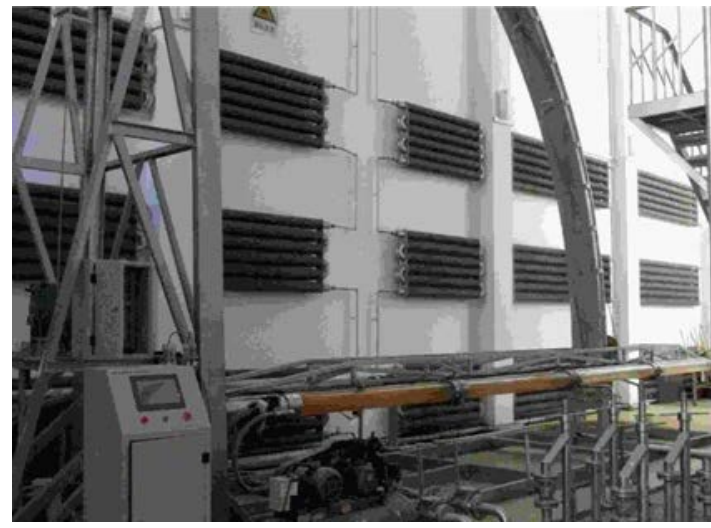

(a)

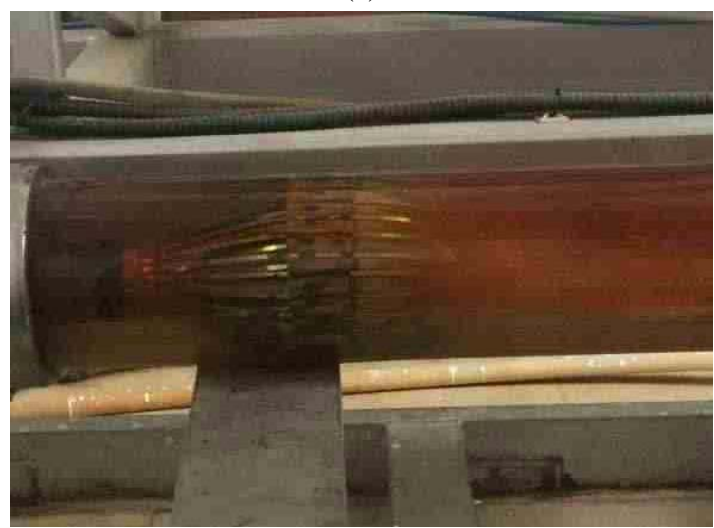

(b)

Fig.15. Horizontal oil-water two-phase flow experiment equipment in petrodaqing test \&experiment center of logging \& testing technology. 
The experimental mediums are tap water, white oil and edible salt. The values of salt concentration $\rho_{w}$ range from $0.5 \mathrm{~g} / \mathrm{L}$ to $5.5 \mathrm{~g} / \mathrm{L}$ which corresponds to the different mineralization degrees of water. The experiments in different mineralization degrees of the fluid are forced into the insulated flow pipe of the SGLCP with $D=20 \mathrm{~mm}$ from a large diameter horizontal plexiglass pipe (the value of inner diameter is $125 \mathrm{~mm}$ ).

\section{A. Static experiments of the SGLCP}

In order to analyze the response characteristics of the SGLCP in the horizontal oil-water segregated flow and to verify the validity and feasibility of obtaining the pure-water electrical conductivity in the condition of a continuous water phase in the segregated flow, the experiments for the $\mathrm{LCP}_{1}$, with $D=20 \mathrm{~mm}, h=2 \mathrm{~mm}, d=1.5 \mathrm{~mm}$ and $S=4,5,6 \mathrm{~mm}$ respectively, in the SGLCP were conducted and the experiments for the horizontal oil-water segregated flow were also carried out, the results of which are shown in Fig.16., under the condition that $\rho_{w}$ is $2.0 \mathrm{~g} / \mathrm{L}$.

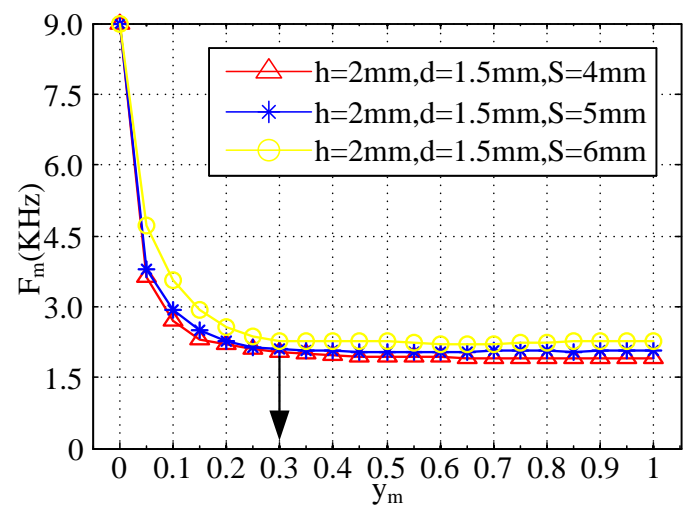

Fig.16. Calibration of the $\mathrm{LCP}_{1}$ with $\rho_{w}=2.0 \mathrm{~g} / \mathrm{L}$ under different $y_{m}$ in the condition of oil-water segregated flow.

As can be seen from Fig.16., under the conditions of higher $y_{m}$, the $\mathrm{LCP}_{1}$ can obtain a reliable and accurate pure-water electrical conductivity, which can be used as the pure-water correction value for water content measurement in horizontal oil water segregated flow. And in the condition of the $\mathrm{LCP}_{1}$ with $D=20 \mathrm{~mm}, h=2 \mathrm{~mm}, d=1.5 \mathrm{~mm}, S=4,5$, $6 \mathrm{~mm}$, respectively, the measured result is approximately equal to pure-water electrical conductivity, i.e., the measurement values of the $\mathrm{LCP}_{1}$ while $y_{m}=1$, when $y_{m}$ is greater than or equal to 0.30 .

Fig.16. shows that under a certain given salt concentration, the SCLGP can obtain the pure-water electrical conductivity at the condition of the high $y_{m}$.

In order to investigate the response characteristics for the different mineralization degrees of water in the fluid to the SGLCP, the different values of the water salt concentrations are used to represent the different mineralization degrees of water during the experiments. The measurement experiments for pure-water electrical conductivity were conducted, and the response values of the experiment are calculated at the condition filled with a continuous water phase in the insulated pipe under different values of $\rho_{w}$ in which the value range of salt concentration $\rho_{w}$ is from 0.5 $\mathrm{g} / \mathrm{L}$ to $5.5 \mathrm{~g} / \mathrm{L}$. Fig.17. shows the calibration response of the SGLCP for pure-water measurement under different values of $\rho_{w}$ in horizontal flow, in which $h=2 \mathrm{~mm}, d=1.5 \mathrm{~mm}$ and $S=4,5,6 \mathrm{~mm}$, respectively.

As can be observed from Fig.17., in different conditions of $\rho_{w}$, the response characteristics of the SGLCP, whose $S$ is set to three different values, are basically consistent. With the increase of $\rho_{w}$, the measured frequency signal $F_{\mathrm{S}}$ of the SGLCP with three different $S$ shows a decreasing tendency and shows obvious difference which can be attributed to the distinctive difference of $\rho_{w}$. Under the conditions of a certain given $\rho_{w}$, the greater the $S$ is, the greater the response result $F_{\mathrm{S}}$ of the SGLCP becomes.

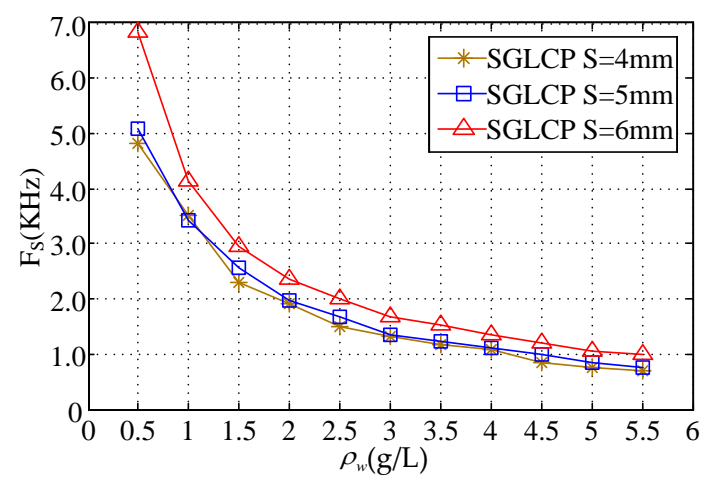

Fig.17. Calibration of the SGLCP under different values of $\rho_{w}$ : $h=2 \mathrm{~mm}, d=1.5 \mathrm{~mm}, S=4,5,6 \mathrm{~mm}$, respectively.

\section{B. Static experiments of the RSCP}

We focused on analyzing static response results of the RSCP with pure-water electrical conductivity measurement under different mineralization degrees. The calculated response results of the RSCP for pure-water measurement are shown Fig.18. As can be observed from Fig.18., with the increase of $\rho_{w}$, the measured frequency $F_{\mathrm{R}}$ of the RSCP shows a decreasing tendency, and shows obvious difference which can be attributed to the distinctive different $\rho_{w}$.

According to Fig.17. and Fig.18., it is found that the SGLCP and RSCP have nice response characteristics for pure-water measurement under different values of $\rho_{w}$, respectively. Taking into account the actual logging in the experiment, the shell of the logging instrument is conductive material and connected with the ground, and the ground terminal of the excitation control circuit in the system driven circuit of the SGLCP and RSCP directly connected with the instrument shell, would result in the shunt or leakage of the current input by the exciting electrodes. The current loss would exhibit a non-linear change with the change of the fluid component or conductivity inside the sensors, causing the non-constant current in the measurement field of the 
sensors, which would cause that the measurement results of SGLCP and RSCP exhibit a non-linear change under different values of $\rho_{w}$.

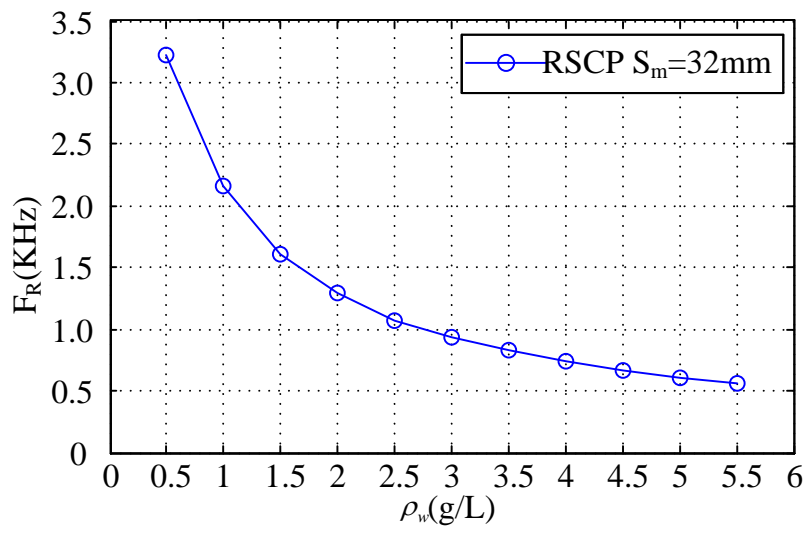

Fig.18. Calibration of the RSCP under different values of $\rho_{w}$ : $h_{\mathrm{e}}=2 \mathrm{~mm}, h_{\mathrm{m}}=2 \mathrm{~mm}, S_{\mathrm{e}}=47 \mathrm{~mm}, \mathrm{c}=32 \mathrm{~mm}$.

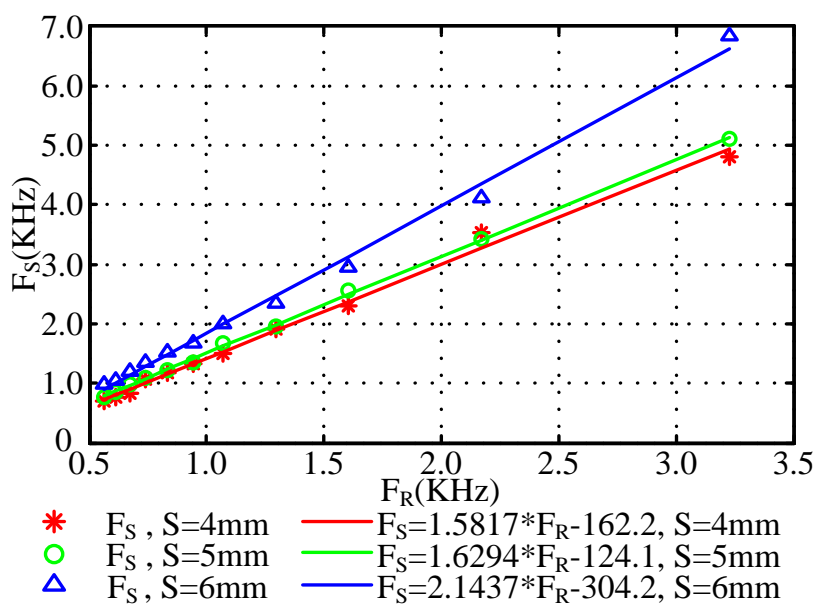

Fig.19. The relation curves of experiment results with the SGLCP and RSCP under different values of $\rho_{w}$.

Fig.19. shows the calibration curves of experiment results between the SGLCP and RSCP. As can be observed from Fig.19., the discrete points with three different marker symbols represent the calibration results of experiment results between the SGLCP and RSCP. The continuous curves with three different colors represent calibration curves of experiment results between the SGLCP and RSCP by using the linear fitting. Under the condition of different $S$, the response values between the SGLCP and RSCP show a relatively good linear function relation, and the calibration curves about the SGLCP and RSCP can be given as

$$
\left\{\begin{array}{l}
F_{\mathrm{S}}=1.5817 F_{\mathrm{R}}-162.2, S=4 \mathrm{~mm} \\
F_{\mathrm{S}}=1.6294 F_{\mathrm{R}}-124.1, S=5 \mathrm{~mm} \\
F_{\mathrm{S}}=2.1437 F_{\mathrm{R}}-304.2, S=6 \mathrm{~mm}
\end{array}\right.
$$

where $F_{\mathrm{R}}$ is the measured frequency of the RSCP, $F_{\mathrm{S}}$ is the measured frequency of the SGLCP, $S$ is the separation between two measuring electrodes of the SGLCP.

In the actual work, the constant current exciting source of the conductance sensors can cause leakage to some degrees. Therefore, some error would be generated for the linear fitting curve of the SGLCP and RSCP.

According to the comprehensive analysis for the simulation and static experiment, the results in Fig.9. and Fig.16. analyze and verify the validity and feasibility of the SGLCP to measure the pure-water electrical conductivity in the condition of high $y_{m}$, and simultaneously, a higher linearity between the SGLCP and RSCP can be verified in terms of the electrical resistivity and salt concentration, respectively. Therefore, the SGLCP plays an important role in the pure-water electrical conductivity for the actual horizontal water content measurement, with the characteristics of low velocity and high water content, and solves the difficult problem that the RSCP cannot obtain the pure-water electrical conductivity of oil water two phase segregated flow in horizontal wells.

\section{ConClusion}

This paper presented SGLCP to measure the pure-water electrical conductivity in horizontal oil-water segregated flow. The simulation models of these structures of the SGLCP and RSCP are established by using the FEM. Simulation experiments with the calculated response of the SGLCP and RSCP measurement area are given, and the electric field distribution under a certain given mineralization degree of water is obtained. It analyzed measurement characteristics and indicated good linearity characteristics between the designed SGLCP and RSCP for pure-water measurement with experiment under different mineralization degrees of water. The validity and feasibility of the designed SGLCP for pure-water electrical conductivity measurement under different mineralization degrees in horizontal oil-water two-phase segregated flow are verified by experiment results. The achievement of this work provides a theoretical basis and technical reference for the research and development of water content measurement instrument and the production profile logging interpretation and evaluation based on horizontal oil-water segregated flow.

\section{ACKNOWLEDGMENT}

This work is supported by Specialized Research Fund for the Doctoral Program of Higher Education of China (20131333110015), Natural Science Foundation of Hebei Province, China (F2014203265, F2015203253), Innovation Foundation for Graduate Students of Hebei Province, China (00302-6370003), Daqing Oilfield Limited Company of Heilongjiang Province, China (DQP-2014-CS-KY-002), and Science and Technology Research \& Development Program of Qinhuangdao City, China (201601B051). 


\section{REFERENCES}

[1] Zhao, X.Q., Zhang, H.F., Pan, B.Z., Zhang, X.F. (2010). Horizontal induction logging influence factors analysis and its application in water-flooded interpretation. Well Logging Technology, 34 (1), 4246.

[2] Kong, L.F., Li, L., Kong, W.H., Liu, X.B., Li, Y.W., Zhang, S.L. (2015). Overview of logging technique in oil production horizontal and high deviated wells. Journal of Yanshan University, 39 (1), 1-8.

[3] Abouelwafa, M.S.A., Kendall, E.J.M. (1980). The use of capacitance sensors for phase percentage determination in multiphase pipelines. IEEE Transactions on Instrumentation and Measurement, 29 (1), 24-27.

[4] Ye, J.M., Peng, L.H., Wang, W.R., Zhou, W.X. (2011). Optimization of helical capacitance sensor for void fraction measurement of gas-liquid two-phase flow in a small diameter tube. IEEE Sensors Journal, 11 (10), 2189-2196.

[5] Zhai, L.S., Jin, N.D., Gao, Z.K., Wang, Z.Y. (2015). Liquid holdup measurement with double helix capacitance sensor in horizontal oil-water two-phase flow pipes. Chinese Journal of Chemical Engineering, 23 (1), 268-275.

[6] Han, L.F. (2008). Study on measurement method of oil-water two-phase flow pattern. Unpublished master dissertation, Tianjin University of Technology, Tianjin, China.

[7] Passey, Q.R., Yin, H., Rendeiro, C.M., Fitz, D.E. (2006). Overview of high-angle and horizontal well formation evaluation: Issues, learning, and future directions. Well Logging Technology, 30 (1), 1-9.

[8] Liu, X.G., Liu, S.Q., Jiang, Z.X. (1998). Horizontal well technology in the oilfield of China. Chemical Communications, 49 (14), 1452-1454.

[9] Du, L.P., Tian, R.F., Sun, Z.N., Liu, X.Y. (2012). Analysis of static response performance of capacitance sensors for measuring humidity of wet steam in air and water systems. Proceedings of the CSEE, 32 (26), 8690.

[10] Wu, H., Tan, C., Dong, X.X., Feng, D. (2015). Design of a conductance and capacitance combination sensor for water holdup measurement in oil-water two-phase flow. Flow Measurement \& Instrumentation, 46, 218229.

[11] Maxwell, J.C. (1882). A Treatise on Electricity and Magnetism. Clarendon Press.

[12] Ceccio, S.L., George, D.L. (1996). A review of electrical impedance techniques for the measurement of multiphase flows. Journal of Fluids Engineering, 118 (118), 391-399.

[13] Liu, X.B. (2000). Research on the methods of multiphase flow well logging and novel sensors. Unpublished doctoral dissertation, China University of Petroleum, Beijing, China.

[14] Asali, J.C., Hanratty, T.J., Paolo, A. (1985). Interfacial drag and film height for vertical annular flow. Aiche Journal, 31 (6), 895-902.
[15] Lucas, G.P., Cory, J.C., Waterfall, R.C. (2000). A sixelectrode local probe for measuring solids velocity and volume fraction profiles in solids-water flows. Measurement Science \& Technology, 11 (11), 14981509.

[16] Devia, F., Fossa, M. (2003). Design and optimisation of impedance probes for void fraction measurements. Flow Measurement \& Instrumentation, 14 (4-5), 139149.

[17] Chao, T., Wang, N.N., Feng, D. (2016). Oil-water two-phase flow pattern analysis with ERT based measurement and multivariate maximum Lyapunov exponent. Journal of Central South University, 23 (1), 240-248.

[18] Trallero, J.L., Sarica, C., Brill, J.P. (1997). A study of oil-water flow patterns in horizontal pipes. SPE Production \& Facilities, 12 (3), 165-172.

[19] Zhai, L.S., Jin, N.D., Zong, Y.B, Wang, Z.Y., Gu, M. (2012). The development of a conductance method for measuring liquid holdup in horizontal oil-water twophase flows. Measurement Science \& Technology, 23 (2), 344-347.

[20] Heng, Z. (1990). Analysis of salt-mud logging interpretation. Chinese Journal of Geophysics, 33 (1), 77-86.

[21] Johansen, G.A., Jackson, P. (2000). Salinity independent measurement of gas volume fraction in oil/gas/water pipe flows. Applied Radiation \& Isotopes, 53 (4-5), 595-601.

[22] Wang, K.W., Sun, J.M., Geng, S.C., Wu, J.L. (2006). Percolation network study of shale effects on rock electrical properties under different salinity. Chinese Journal of Geophysics, 49 (6), 1710-1717.

[23] Sætre, C., Johansen, G.A., Tjugum, S.A. (2010). Salinity and flow regime independent multiphase flow measurements. Flow Measurement \& Instrumentation, 21 (4), 454-461.

[24] Wang, Y.J., Hu, J.H., Huang, C.H., Li, L., Liu, X.B. (2010). Adaptive modules design of impedance watercut meter and its applications. Well Logging Technology, 34 (5), 469-472.

[25] Ji, P.C., Tan, C., Dong, F. (2013). Field characteristic of two-phase flow measurement based on long waist cone and conductance sensor fusion. Journal of Central South University, 44 (S1), 415-419.

[26] Deng, X., Yang, W. (2012). Fusion research of electrical tomography with other sensors for two-phase flow measurement. Measurement Science Review, 12 (2), 62-67.

[27] Mo, H., Deng, J.Z. (2006). Field Theory. Beijing, China: Atomic Energy Press.

[28] Zhou, C.Z. (1965). Composition Analysis Instrument (Vol. 1). Beijing, China: Mechanical Industry Press.

Received July 03, 2016 Accepted August 10, 2016. 